\title{
INDEPENDENT CALIBRATION OF WATER VAPOR RAMAN LIDAR BY USING ADDITIONAL ELASTIC MEASUREMENTS AT WATER VAPOR RAMAN WAVELENGTH
}

\author{
Decheng Wu ${ }^{1} 2^{*}$, Zhien Wang ${ }^{1}$, Dong Liu ${ }^{2}$, Chenbo Xie ${ }^{2}$, Yingjian Wang ${ }^{2}$ \\ ${ }^{1}$ Department of Atmospheric Sciences, University of Wyoming, WY 82070, USA, \\ ${ }^{2}$ Hefei Institutes of Physical Science, Chinese Academy of Sciences, Anhui 230031, China
}

*Email:dwu5@uwyo.edu

\begin{abstract}
A new method for independent calibration of water vapor Raman lidar by using additional measurements of elastic signals at water vapor Raman wavelength was introduced. The water vapor mixing ratio calibration constant was derived from nitrogen mixing ratio calibration constant and Rayleigh to water vapor Raman backscattering cross section ratio. The nitrogen mixing ratio calibration constant with uncertainties of 1 2\% can be determined from the nitrogen mixing ratio measurement in an aerosolfree zone in the upper troposphere with the additional elastic measurement and nitrogen Raman measurement, the Rayleigh to water vapor Raman backscattering cross section ratio can be calculated with uncertainties of about $8 \%$, or can be determined experimentally with uncertainties of less than $5 \%$. Using the experimental cross section ratio, a total uncertainty of the water vapor calibration less than $6 \%$ is possible to achieve. Because this calibration method utilizes actual lidar signals in nitrogen and water vapor detection channels, the calibration can fully capture lidar state changes.
\end{abstract}

\section{INTRODUCTION}

Water vapor mixing ratio (WVMR) calibration is an important issue for water vapor Raman lidar (WVRL), in particular for climate monitoring applications [1]. The calibration usually relies on an external, independent measurement of water vapor, such as radiosonde or microwave radiometer. The calibration accuracy relies on accuracies of these independent measurements and synchronization of the measurements [2 3]. As the changes of the WVRL state, such as changes in overall system efficiency and detector sensitivity due to ageing, will influence the calibration constant, a regular calibration is needed for a WVRL [1, 3]. Therefore, it is difficult to obtain long-term regularly independent water vapor measurements at most of WVRL sites.

In general, WVRL calibrations need to determine the difference of system detection efficiencies between the nitrogen and water vapor channels and nitrogen to water vapor Raman backscattering cross section ratio. Using a calibrated tungsten lamp, the ratio of lidar channel detection efficiencies could be calibrated with errors of about $1 \%$ [4]. The backscattering cross section ratio of nitrogen to water vapor is needed in the lamp independent calibration, the cross section ratio can be calculated with uncertainties of about $\pm 10 \%$, or can be determined experimentally with an error of less than $5 \%$ based on a well-calibrated WVRL $[4,5]$. But the cross section ratio is system dependent due to different filters used. However, there are several serious limitations in the lamp independent calibration due to not able to fully capture lidar state changes [6].

In this paper, a new independent calibration method for WVRL based on additional elastic measurement at water vapor Raman wavelength was introduced and discussed.

\section{METHODOLOGY}

The measured Raman signals from nitrogen and water vapor molecules can be written as below.

$$
\begin{aligned}
V_{N}\left(z, \lambda_{N}\right)= & E_{L} \frac{c}{2} A \eta_{N} \frac{1}{z^{2}} N_{N}(z) \sigma_{N, \pi}\left(\lambda_{L}, \lambda_{N}\right) \\
& T\left(z, z_{0}, \lambda_{L}\right) T\left(z, z_{0}, \lambda_{N}\right) \\
V_{w v}\left(z, \lambda_{w v}\right)= & E_{L} \frac{c}{2} A \eta_{w v} \frac{1}{z^{2}} N_{w v}(z) \sigma_{w v, \pi}\left(\lambda_{L}, \lambda_{w v}\right) \\
& T\left(z, z_{0}, \lambda_{L}\right) T\left(z, z_{0}, \lambda_{w v}\right)
\end{aligned}
$$

where, $\mathrm{z}$ is the altitude of scattering volume; $z_{0}$ is the altitude of lidar; $\mathrm{V}_{\mathrm{N}}\left(\mathrm{z}, \lambda_{\mathrm{N}}\right)$ and $\mathrm{V}_{\mathrm{wv}}\left(\mathrm{z}, \lambda_{\mathrm{wv}}\right)$ are 
the voltage values of nitrogen and water vapor Raman signals without background signals in nitrogen and water vapor channels; $\lambda_{\mathrm{L}}$ is the laser wavelength; $\lambda_{N}$ and $\lambda_{\mathrm{wv}}$ are the nitrogen and water vapor Raman shifted wavelength; $E_{L}$ is the laser pulse energy; $\mathrm{c}$ is the light speed; $\mathrm{A}$ is the effective telescope receiving area; $\eta_{\mathrm{N}}$ and $\eta_{\mathrm{wv}}$ are the total efficiencies of nitrogen and water vapor Raman channels, including optical efficiency, quantum efficiency, and electrical efficiency; $\mathrm{N}_{\mathrm{N}}(\mathrm{z})$ and $\mathrm{N}_{\mathrm{wv}}(\mathrm{z})$ are the nitrogen and water vapor molecular number densities at $\mathrm{z} ; \sigma_{\mathrm{N}, \pi}\left(\lambda_{\mathrm{L}}, \lambda_{\mathrm{N}}\right)$ and $\sigma_{\mathrm{wv}, \pi}\left(\lambda_{\mathrm{L}}, \lambda_{\mathrm{wv}}\right)$ are nitrogen and water vapor Raman backscattering cross sections; $\mathrm{T}\left(\mathrm{z}, \mathrm{z}_{0}, \lambda_{\mathrm{N}}\right)$ and $\mathrm{T}(\mathrm{z}$, $\left.\mathrm{z}_{0}, \lambda_{\mathrm{wv}}\right)$ are the atmospheric transmissions from $\mathrm{z}_{0}$ to $\mathrm{z}$ at the wavelength of $\lambda_{\mathrm{N}}$ and $\lambda_{\mathrm{wv}}$.

Temperature influence on the backscattering cross section was not considered in lidar equations in this section, it will be discussed in section 3. Lidar signals in the range of full overlap were selected for calibration to avoid uncertainties caused by the overlap function. Therefore, the overlap functions were neglected in lidar equations in this paper.

Water vapor mixing ratio $\mathrm{w}(\mathrm{z})$ is derived from the ratio of water vapor to nitrogen Raman signals,

$w(z)=C_{w v} \frac{V_{w v}\left(z, \lambda_{w v}\right)}{V_{N}\left(z, \lambda_{N}\right)} \frac{T\left(z, z_{0}, \lambda_{N}\right)}{T\left(z, z_{0}, \lambda_{w v}\right)}$

where, $\mathrm{C}_{\mathrm{wv}}$ is the WVMR calibration constant.

$C_{w v}=0.485 \frac{\eta_{N}}{\eta_{w v}} \frac{\sigma_{N, \pi}\left(\lambda_{L}, \lambda_{N}\right)}{\sigma_{w v, \pi}\left(\lambda_{L}, \lambda_{w v}\right)}$

After WVMR measurement, extra laser pulses at a wavelength $\lambda_{\mathrm{C}}$, which is very close to $\lambda_{\mathrm{wv}}$, were emitted into the atmosphere. If the difference between $\lambda_{\mathrm{C}}$ and $\lambda_{\mathrm{wv}}$ is less than the half of the filter bandwidth in water vapor channel $\Delta \lambda_{\mathrm{wv}}, \mid \lambda_{\mathrm{C}^{-}}$ $\lambda_{\text {wv }}<\Delta \lambda_{\text {wv }} / 2$, the elastic signal from the atmosphere at the wavelength $\lambda_{\mathrm{C}}$ can be measured by using the water vapor channel.

$$
\begin{aligned}
V_{C}\left(z, \lambda_{C}\right)= & E_{C} \frac{c}{2} A \eta_{w v} \varepsilon\left(\lambda_{C}, \lambda_{w v}\right) \frac{1}{z^{2}} \\
& {\left[\beta_{a}\left(z, \lambda_{C}\right)+N_{a i r}(z) \sigma_{m, \pi}\left(\lambda_{C}\right)\right] } \\
& {\left[T\left(z, z_{0}, \lambda_{C}\right)\right]^{2} }
\end{aligned}
$$

where, $V_{C}\left(z, \lambda_{C}\right)$ is the voltage value of elastic signal without background signal received by the water vapor channel; $\varepsilon\left(\lambda_{\mathrm{C}}, \lambda_{\mathrm{wv}}\right)$ is the ratio of total efficiency of the water vapor channel at $\lambda_{c}$ to the one at $\lambda_{\mathrm{wv}}$, the difference between $\lambda_{\mathrm{C}}$ and $\lambda_{\mathrm{wv}}$ is so little that $\varepsilon\left(\lambda_{\mathrm{C}}, \lambda_{\mathrm{wv}}\right)$ is mainly determined by the narrow bandwidth filter in the water vapor channel; $\beta_{\mathrm{a}}\left(\mathrm{z}, \lambda_{\mathrm{C}}\right)$ is the aerosol backscattering coefficient at $\lambda_{C} ; \mathrm{N}_{\text {air }}(\mathrm{z})$ is the air molecular number density; $\quad \sigma_{\mathrm{m}, \pi}\left(\lambda_{\mathrm{C}}\right) \quad$ is Rayleigh backscattering cross section at $\lambda_{\mathrm{C}} ; \mathrm{T}\left(\mathrm{z}, \mathrm{z}_{0}, \lambda_{\mathrm{C}}\right)$ is the atmospheric transmission from $\mathrm{z}_{0}$ to $\mathrm{z}$ at $\lambda_{\mathrm{C}}$, $\mathrm{T}\left(\mathrm{z}, \mathrm{z}_{0}, \lambda_{\mathrm{C}}\right) \approx \mathrm{T}\left(\mathrm{z}, \mathrm{z}_{0}, \lambda_{\mathrm{wv}}\right)$.

In the free troposphere, an aerosol-free zone, where the contribution of aerosol backscattering to the elastic signal could be neglected, can be often found. In the aerosol-free zone, the elastic signal measured by using water vapor channel could be written as below,

$$
\begin{aligned}
V_{C}\left(z, \lambda_{C}\right)= & E_{C} \frac{c}{2} A \eta_{w v} \varepsilon\left(\lambda_{C}, \lambda_{w v}\right) \frac{1}{z^{2}} N_{a i r}(z) \sigma_{m, \pi}\left(\lambda_{C}\right) \\
& {\left[T\left(z, z_{0}, \lambda_{C}\right)\right]^{2} }
\end{aligned}
$$

In the aerosol-free zone, nitrogen mixing ratio (NMR), which is a constant in the atmosphere below about $100 \mathrm{~km}$, could be derived from the ratio of nitrogen Raman signal in Eq. (1) to Rayleigh signal in Eq. (6).

$$
\begin{aligned}
\operatorname{NMR}(z) & =0.755 \\
& =C_{N} \frac{E_{C}}{E_{L}} \frac{V_{N}\left(z, \lambda_{N}\right)}{V_{C}\left(z, \lambda_{C}\right)} \frac{\left[T\left(z, z_{0}, \lambda_{C}\right)\right]^{2}}{T\left(z, z_{0}, \lambda_{L}\right) T\left(z, z_{0}, \lambda_{N}\right)}
\end{aligned}
$$

where, $C_{N}$ is the NMR calibration constant.

$C_{N}=0.967 \varepsilon\left(\lambda_{C}, \lambda_{w v}\right) \frac{\eta_{w v}}{\eta_{N}} \frac{\sigma_{m, \pi}\left(\lambda_{C}\right)}{\sigma_{N, \pi}\left(\lambda_{L}, \lambda_{N}\right)}$

Using Eq. (7), $C_{N}$ could be determined in the aerosol-free zone. $\mathrm{C}_{\mathrm{wv}}$ could be derived from the determined $\mathrm{C}_{\mathrm{N}}$ and Rayleigh to water vapor backscattering cross section ratio by using Eq. (9) shown below.

$$
C_{w v}=0.469 \frac{1}{C_{N}} \varepsilon\left(\lambda_{C}, \lambda_{w v}\right) \frac{\sigma_{m, \pi}\left(\lambda_{C}\right)}{\sigma_{w v, \pi}\left(\lambda_{L}, \lambda_{w v}\right)}
$$

\section{DISCUSSIONS}

\subsection{Rayleigh Backscattering Signal}

Measuring Rayleigh backscattering signals by using the water vapor channel is the key in the proposed method.

Firstly, an extra laser source is needed, and its output wavelength should be very close to the filter central wavelength (CWL) in the water vapor channel. For the most of WVRLs, the filter CWL in the water vapor channel is about 407.5 
nm. OPO based on a type II BBO crystal can generate narrow linewidth nanosecond laser pulse at the wavelength of $407.5 \mathrm{~nm}$. The nanosecond laser pulse at $354.7 \mathrm{~nm}$ employed in the most of WVRLs, can be used to pump the OPO to get the needed wavelength.

Secondly, an aerosol-free zone should be found. When the efficiency of the OPO is $5 \%$, the intensity of the Rayleigh signal at the height of 10 $\mathrm{km}$ approximately equals the water vapor Raman signal at the height of $1 \mathrm{~km}$ with a WVMR of 10 $\mathrm{g} / \mathrm{kg}$ under a fair atmosphere condition. Therefore, the elastic signal from the upper troposphere with the OPO laser could be detected by the water vapor channel under the cloud-free atmosphere condition. Because a high pulse energy laser and a large telescope are employed in ground-based WVRLs and temporal averaging is normally used in the data processing, the averaged nitrogen Raman signal is quite smooth in the upper troposphere, even up to the tropopause. In summary, an aerosol-free zone could be measured by the proposed system in the upper troposphere.

In the aerosol-free zone, backscattering ratio at $407.5 \mathrm{~nm}$ should be less than 1.01, and the error cause by the aerosol-free assumption will be less than $1 \%$. To reduce the noise level, the calibration would be carried out during nighttime. Due to temporal averaging in data processing, the error of laser energy correction could be neglected.

\subsection{Backscattering Cross Sections and Temperature Dependence}

Nitrogen and water vapor Raman backscattering cross sections, as well as Rayleigh backscattering cross section, are involved in this method.

With filter bandwidths of $0.3 \mathrm{~nm}$, calculated temperature-dependent effective nitrogen Raman backscattering cross section at $386.7 \mathrm{~nm}$ and Rayleigh backscattering cross section at $407.5 \mathrm{~nm}$, as well as the ratio of Rayleigh to nitrogen Raman backscattering cross section, were shown in figure 1. The relative difference between Rayleigh to nitrogen Raman effective backscattering cross section ratios at $320 \mathrm{~K}$ and $200 \mathrm{~K}$ is about $0.26 \%$, therefore, the temperature influence could be neglected in the determination of $\mathrm{C}_{\mathrm{N}}$.

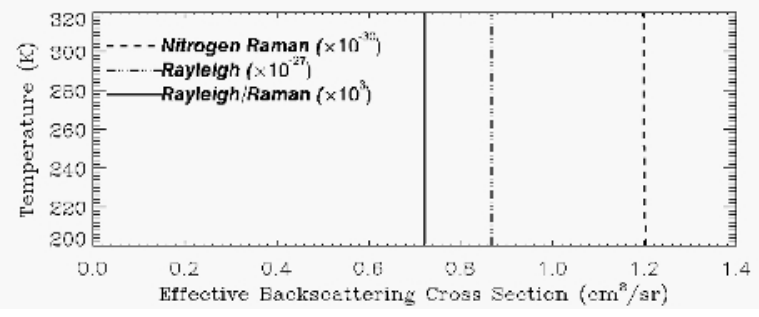

Figure 1. Calculations of temperature-dependent effective nitrogen Raman backscattering cross section at $386.7 \mathrm{~nm}$ (dash), Rayleigh

backscattering cross section at $407.5 \mathrm{~nm}$ (dash

dot), and the ratio of Rayleigh to nitrogen Raman

effective backscattering cross section (solid).

In the determination of $C_{w v}$ using Eq. (9), temperature-dependent Rayleigh to water vapor Raman backscattering cross section ratio is needed. The error of theoretical calculation of Rayleigh scattering cross section is less than $1 \%$ [7], and the error of calculated water vapor absolute Raman differential scattering cross section of the Q branch of $v 1$ is less than 8\% [8]. However, the cross section ratio could be determined experimentally if the lidar could point horizontally to provide $\mathrm{C}_{\mathrm{wv}}$ by comparing lidar measurements with accurate in situ measurements on the laser path. Then with Eq. (9), the cross section ratio can be determined with accuracy better than $5 \%$ for future vertical pointing measurements. The temperature-dependence of the cross section ratio could be calculated with uncertainties of about $1 \%$ [4].

\subsection{Atmospheric Transmission Correction}

As shown in Eq. (7), two-way transmission of the atmosphere between lidar and the aerosol-free zone needs to be corrected in the determination of $\mathrm{C}_{\mathrm{N}}$. The two-way transmission corrections calculated by using different aerosol Angstrom exponents $\mathrm{k}$ under different aerosol optical depth (AOD) conditions were shown in figure 2. From figure 2, it can be seen that the error of atmospheric transmission correction caused by the uncertainty of $\mathrm{k}$ increases along with the increasing AOD, so the calibration should be carried out under low aerosol loading conditions. $\mathrm{AOD}$ and $\mathrm{k}$ can be retrieved from the elastic signals at $354.7 \mathrm{~nm}$ and $407.5 \mathrm{~nm}$ and Raman signal at $386.7 \mathrm{~nm}$ measured by the lidar during the calibration experiment. Even for AOD at 355 
$\mathrm{nm}$ equals $0.2,20 \%$ uncertainty in $\mathrm{k}$ yields about $0.6 \%$ uncertainty in transmission correction.

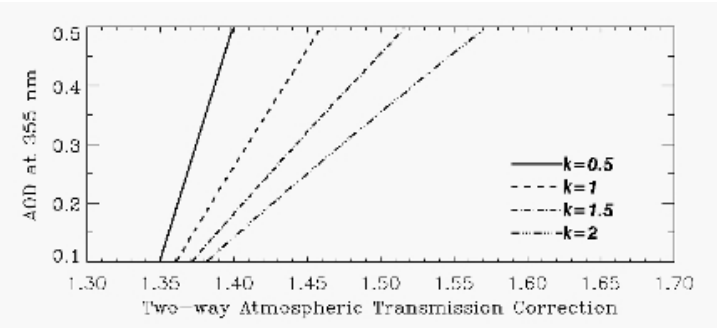

Figure 2. Calculated two-way atmospheric transmission corrections in NMR in Eq. (7) as a function of AOD by using different Angstrom exponent $\mathrm{k}$.

\section{CONCLUSIONS}

In this paper, a new independent calibration method for WVRL was proposed based on additional elastic measurements at water vapor Raman wavelength. The water vapor mixing ratio calibration constant was derived from the nitrogen mixing ratio calibration constant and Rayleigh to water vapor Raman backscattering cross section ratio.

Nitrogen mixing ratio calibration constant indicates difference of total efficiencies between the nitrogen and water vapor channels. The error of the determined nitrogen mixing ratio calibration constant is about $1 \sim 2 \%$. The Rayleigh to water vapor Raman backscattering cross section ratio can be calculated with uncertainties of about $8 \%$. The cross section ratio also can be determined experimentally from a well-calibrated WVRL with uncertainties of less than 5\%. Using the experimental cross section ratio, the total error of the water vapor calibration will be less than $6 \%$.

Compared with the lamp calibration method, this new approach can fully capture the changes of lidar state. Furthermore, after the initial design and integration, the calibration can be done routinely and automatically.

\section{ACKNOWLEDGEMENTS}

This research work is supported by NSFC under grant No.41405032 and partly supported by NSF under AGS-1337599.

\section{REFERENCES}

[1] Commission for instruments and methods of observation, 2013: Expert team on new technologies and testbeds first section final report, Geneva, Switzerland, 4-7 March.

[2] Davide Dionisi, Fernando Congeduti, Gian Luigi Liberti, Francesco Cardillo, 2010: Calibration of a multichannel water vapor Raman lidar through noncollocated operational soundings: optimization and characterization of accuracy and variability, J. Atmos. Oceanic Technol., 27, 108-121.

[3] T. Leblanc, I. S. McDermid, 2008: Accuracy of Raman lidar water vapor calibration and its applicability to long-term measurements, Appl. Opt., 47(30), 5592-5603.

[4] Whiteman, D. N, T. Berkoff, D. D. Turner, T. P. Tooman, R. A. Ferrare, and L. A. Heilman, 2000: Research efforts in the absolute calibration of a Raman water vapor lidar, Proc. of the $20^{\text {th }}$ International Laser Radar Conference, Vichy, France.

[5] D. D. Venable, D. N. Whiteman, M. N. Calhoun, A. O. Dirisu, R. N. Connell, and E. Landulfo, 2011: A lamp mapping technique for independent determination of the water vapor mixing ratio calibration factor for a Raman lidar system, Appl. Opt., 50(23), 4622-4632.

[6] D. N. Whiteman, D. D. Venable, and E. Landulfo, 2011: Comments on: Accuracy of Raman lidar water vapor calibration and its applicability to long-term measurements, Appl. Opt., 50(15), 2170-2176.

[7] R. Thalman, K. J. Zarzana, M. A. TOLBERT, R. Volkamer, 2014: Rayleigh scattering cross-section measurements of nitrogen, argon, oxygen and air, JQSRT, 147, 171-177.

[8] G. Avila, J. M. Fernández, G. Tejeda, S. Montero, 2004: The Raman spectra and cross-sections of HO, DO, and HDO in the OH/OD stretching regions, $J$. Mol. Spectrosc., 228, 38-65. 